\title{
Accurate Displacement Detection with a Computer-Aided Interferometric Measuring System
}

\author{
V. Gökhan BöcekÇI* and H. SelÇUK Varol \\ Department of Electronic-Computer Education, University of Marmara, 34722 İstanbul, Turkey \\ In this study, real time measurement of the linear expansion of industrial material undergoing temperature \\ change by using a computer-aided interferometric measuring technique with micrometer accuracy is attempted. \\ Aluminum and copper were subjected to temperature change in the thermal expansion experiment unit (LETI-1) \\ causing them to expand linearly. The fringe number in the interference symbol, which includes the information \\ about linear expansion and temperature change were transferred to a computer via a data acquisition card, \\ graphed by a program created in the LabVIEW environment, and the amount of linear expansion was detected in \\ real time.
}

PACS: 06.30.Bp, 42.87.Bg, 42.62.Cf

\section{Introduction}

Being able to make correct and accurate small displacement measurements, an important industrial application has great importance in terms of acquiring the most precision in the measurement of expansion and shrinkage of industrial materials. In addition, as the demand for detailed information about the dimensional stability and thermal expansion of advanced technological materials has increased day by day, the accuracy of measurements is continually being improved [1].

Laser interferometers are widely used due to their convenience in obtaining submicron resolution in measurements of linear displacement, such as semiconductor metrology and lithography [2-5]. Interferometric apparatus, in which the interference characteristics of light are utilized to be able to make accurate measurements, are preferred because the area to be measured and the measurement apparatus are not supposed to touch each other. Interferogram interference patterns including data, obtained from an interferometric measurement system, can be analyzed by electronic circuits or by ana$\log$ /digital converters.

Suska measured the linear thermal coefficient of expansion of stick-shaped materials $100-1300 \mathrm{~mm}$ in length and $60 \times 40 \mathrm{~mm}^{2}$ in diameter between the temperatures of 10 and $40^{\circ} \mathrm{C}[6]$.

Having measured the expansion amount of copper at four different temperatures $\left(a=21.3^{\circ} \mathrm{C}, b=25.7^{\circ} \mathrm{C}\right.$, $c=31.6^{\circ} \mathrm{C}$, and $d=40.7^{\circ} \mathrm{C}$ ) by using a laser interferometer, Zagar tested the linearity of the system using the equation $\Delta a b+\Delta b c+\Delta c d=\Delta a d[7]$.

* corresponding author; e-mail: vgbocekci@marmara.edu.tr
The aim of this study is to measure the linear expansion of aluminum and copper undergoing temperature change with a computer-aided measuring system having micrometer accuracy.

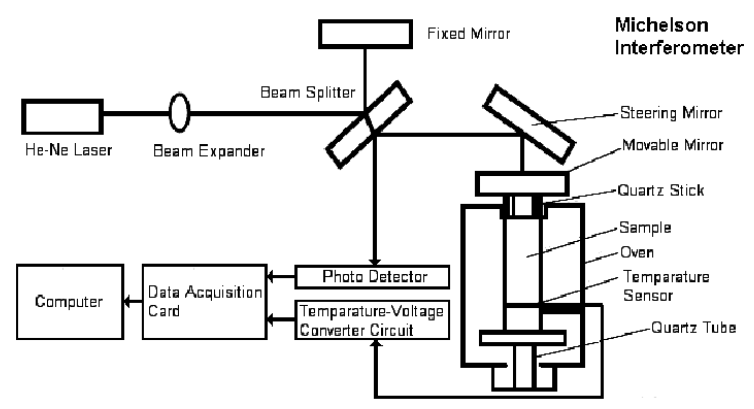

Fig. 1. The computer-aided interferometric measurement system.

The thermal expansion experiment unit LETI-1, based upon the principle of the Michelson interferometer measurement system as shown in Fig. 1, consists of photodetector circuits used in detecting the bright and dark interference fringes, the temperature-voltage converter circuit for converting the instantaneous temperature values of the subject material measured between $0-127.5^{\circ} \mathrm{C}$ into voltage values between $0-10 \mathrm{~V}$, the IOtech personal $\mathrm{DAQ} / 55$ usb data acquisition card used in transferring these values to the computer, and the program created in LabVIEW 8.2, used in analyzing the data and calculating the linear expansion amount. In addition, the amount of linear expansion resulting from the instantaneous temperature changes could be monitored via the program interface. 


\section{Fundamentals of interferometry}

The main components of the Michelson interferometer used in this study are seen in Fig. 2.

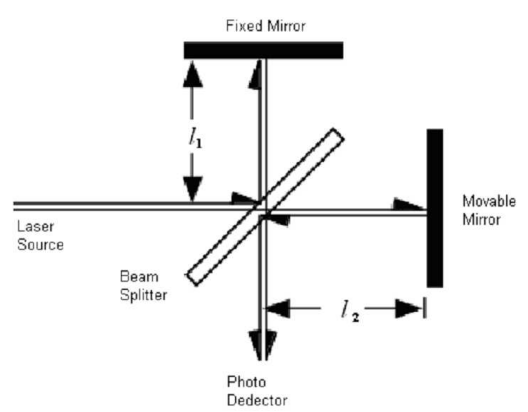

Fig. 2. Main components of the Michelson interferometer.

In this system, the beam emanating from the laser source is divided into two by the beam divider. One of the beams reaches the fixed reflector mirror and the other reaches the moving mirror. When a beam having the electric field component with amplitude $E_{0}$ is divided by the beam divider and reunites then the intensity $I$ of the beam takes the form in Eq. (1):

$$
I=E_{0}^{2}\left[1+\cos 2\left(l_{1}-l_{2}\right) \frac{2 \pi}{\lambda}\right] .
$$

Here, $l_{1}$ represents the distance between the beam-splitter and the fixed mirror, $l_{2}$ represents the distance between the beam-splitter and the moving mirror, $\lambda$ represents the wavelength of the beam.

When the moving mirror travels the distance $l_{1}-l_{2}=$ $\Delta L$ because of the expansion of the subject material, the intensity of the light changes depending on Eq. (1). If $\Delta L$ changes by $\lambda / 2$ or its multiples, light maxima occur. If it changes by $\lambda / 4$ or its multiples, light minima occur. Bright and dark fringes in the interference pattern shift because of the displacement of the moving mirror. By finding the number of fringes passing over the photodetector, total displacement is determined using Eq. (2):

$$
\Delta L=\frac{\lambda}{2} N
$$

Here, $\Delta L$ is the amount of displacement, $\lambda$ is the wavelength of the laser beam, and $N$ is the number of fringes passing over the photodetector.

\section{Experimental study}

In the study we carried out, pieces of aluminium and copper with dimensions of $150 \times 30 \mathrm{~mm}^{2}$ were used as subject materials. The thermal expansion factors of the subject materials are $22.7 \times 10^{-6} /{ }^{\circ} \mathrm{C}$ and $20.6 \times 10^{-6} /{ }^{\circ} \mathrm{C}$, respectively.

The linear expansion of the subject materials placed in the oven of the thermal expansion experiment unit

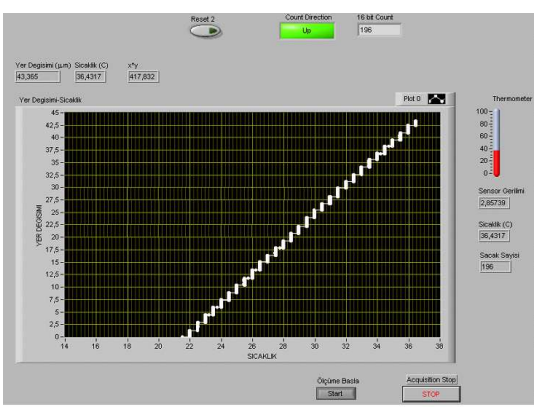

Fig. 3. The displacement-temperature graph interface created in LabVIEW 8.2.

LETI-1 is achieved by increasing the interior oven temperature. When the linearly expanding subject material changes the location of the mirror fixed to itself by half of the wavelength $(\lambda=632.8 \mathrm{~nm})$ of the $\mathrm{He}-\mathrm{Ne}$ laser the result is the shift of the interference fringes. The signal containing the number of the shifted interference fringes is detected by the photodetector and transferred to the data acquisition card to be analyzed in LabVIEW 8.2. The amount of displacement is calculated as indicated in Eq. (2) after the number of fringes is determined. The temperature values of the metals are transferred to the temperature-voltage converter circuit by the temperature sensor, through the data acquisition card and onto the LabVIEW 8.2 software. The displacementtemperature graph can be monitored in real time via the interface (Fig. 3) created in LabVIEW 8.2.

\section{Results and discussion}

The curve fitting method was applied to the measurement results to determine the characteristic of the measurement system. The displacement $(\mu \mathrm{m})-$ temperature $\left({ }^{\circ} \mathrm{C}\right)$ graph for copper is shown in Fig. 4 .

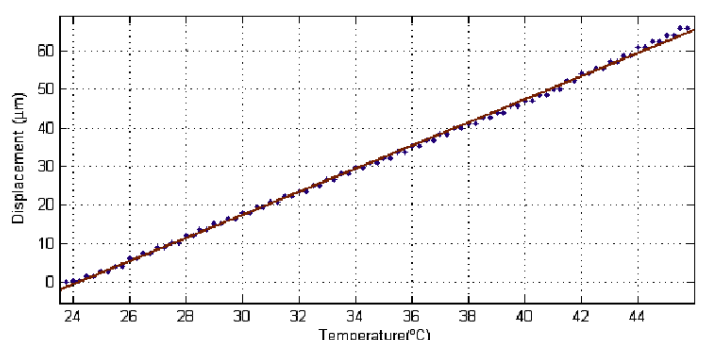

Fig. 4. The displacement-temperature graph for copper.

Whereas the theoretical linear expansion for copper is calculated as $67.98 \mu \mathrm{m}$, the linear expansion amount determined by Eq. (2) was $65.71 \mu \mathrm{m}$ as a result of the measurement. The displacement $(\mu \mathrm{m})$-temperature $\left({ }^{\circ} \mathrm{C}\right)$ graph for aluminum is seen in Fig. 5.

Whereas the theoretical linear expansion for aluminum is calculated as $68.10 \mu \mathrm{m}$, the linear expansion amount 


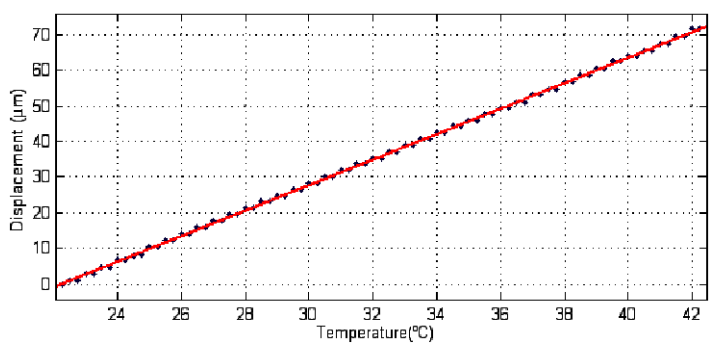

Fig. 5. The displacement-temperature graph for aluminum.

determined by Eq. (2) was $71.68 \mu \mathrm{m}$ as a result of the measurement.

\section{Conclusion}

A computer-aided measurement system via which changes in displacement as temperature varies can be monitored in real time was realized in our study. Results with micrometer accuracy can be obtained with this measurement system, in which a laser interferometer is used. As a result of the experiments carried out with copper and aluminum, it was confirmed that the measurement system is linear by utilizing the MatLab curve-fitting tool.

\section{References}

[1] R. Schödel, Measur. Sci. Technol. 19, 8 (2008).

[2] N. Bobroff, Measur. Sci. Technol. 9, 907 (1993).

[3] S. Donati, L. Falzoni, S. Merlo, IEEE Trans. Instrum. Measur. 45, 942 (1996).

[4] J. Lawall, E. Kessler, Rev. Sci. Instrum. 71, 2669 (2000).

[5] J.H. Yi, J.A. Kim, S. Kim, Y.K. Kwak, Institute Electr. Electron. Eng. 2, 723 (2000).

[6] J. Suska, J. Tschirnich, Measur. Sci. Technol. 10, 55 (1999).

[7] B.G. Zagar, IEEE Trans. Instrum. Measur. 43, 332 (1994). 